\title{
Predictive scotogenic model with flavor dependent symmetry
}

\author{
Zhi-Long Han ${ }^{1, \mathrm{a}} \mathbb{D}$, Weijian Wang ${ }^{2, \mathrm{~b}}$ \\ ${ }^{1}$ School of Physics and Technology, University of Jinan, Jinan 250022, Shandong, China \\ ${ }^{2}$ Department of Physics, North China Electric Power University, Baoding 071003, China
}

Received: 28 March 2019 / Accepted: 8 June 2019 / Published online: 18 June 2019

(C) The Author(s) 2019

\begin{abstract}
In this paper, we propose a viable approach to realise two texture-zeros in the scotogenic model with flavor dependent $U(1)_{B-2 L_{\alpha}-L_{\beta}}$ gauge symmetry. These models are extended by two right-handed singlets $N_{R i}$ and two inert scalar doublets $\eta_{i}$, which are odd under the dark $Z_{2}$ symmetry. Among all the six constructed textures, texture $A_{1}$ and $A_{2}$ are the only two allowed by current experimental limits.

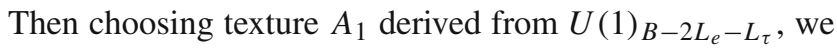
perform a detail analysis on the corresponding phenomenology such as predictions of neutrino mixing parameters, lepton flavor violation, dark matter and collider signatures. One distinct nature of such model is that the structure of Yukawa coupling $\bar{L} \tilde{\eta} N_{R}$ is fixed by neutrino oscillation data, and can be further tested by measuring the branching ratios of charged scalars $\eta_{1,2}^{ \pm}$.
\end{abstract}

\section{Introduction}

It is well known that the standard model (SM) needs extensions to accommodate two missing spices: the tiny but nozero neutrino masses and the cosmological dark matter (DM) candidates. One way of incorporating above two issues in a unified framework is the scotogenic model [1-3], where neutrinos are radiatively generated and the DM field serves as intermediate messengers propagating inside the loop diagram. With all new particles around $\mathrm{TeV}$ scale, the scotogenic model leads to testable phenomenologies [4-31]. Therefore, viable models are extensively studies in recent years [32].

On the other hand, the understanding of the leptonic flavor structure is still one of the major open questions in particle physics. The consensus is that the leptonic mass texture is tightly restricted under the present experimental data. An attractive approach is to consider two texture-zeros in neu-

\footnotetext{
a e-mail: sps_hanzl@ujn.edu.cn

b e-mail: wjnwang96@aliyun.com
}

trino mass matrix $\left(M_{v}\right)$ so that the number of parameters in the Lagrangian is reduced [33]. The phenomenological analysis of two texture-zeros models have been studied in Refs. $[34,35]$. Among fifteen logically patterns, seven of them are compatible to the low-energy experimental data.

On the theoretical side, the simplest way of realizing texture-zeros is to impose the discrete $Z_{N}$ flavor symmetry [36]. However, it might be more appealing to adopt gauge symmetries instead of discrete ones, because the latter may be treated as the residual of $U(1)$ gauge symmetry. It is noted that one can not set any restriction on lepton mass matrix by means of fields with flavor universal charges. Thus the flavor dependent $U(1)$ gauge symmetry is the reasonable choice. Along this thought of idea, specific models are considered in the context of seesaw mechanisms. In Ref. [37], the two texture-zeros are realized based on the anomaly-free $U(1)_{X}$ gauge symmetry with $X \equiv B-\sum x_{\alpha} L_{\alpha}(\alpha=e, \mu, \tau)$ being the linear combination of baryon number $B$ and the lepton numbers $L_{\alpha}$ per family. In Ref. [38], more solutions are found in the type-I and/or III seesaw framework.

It is then natural to ask, if predictive texture-zeros in $M_{v}$ can be realized in the scotogenic scenario and several attempts have been made in this direction. For example, one texture-zero is recently considered in Ref. [39]. Texture $B_{1}-B_{4}$ have been discussed in a model-independent way in Ref. [40]. Texture $C$ is obtained by introducing $U(1)_{L_{\mu}}-L_{\tau}$ gauge symmetry [41-44]. Texture $B_{2}$ is realised with $U(1)_{L_{e}+L_{\mu}-L_{\tau}}$ gauge symmetry in Ref. [45]. If the quark flavor is also flavor dependent, e.g., $U(1)_{x} B_{3}-x L_{e}-L_{\mu}+L_{\tau}$, then one can further interpret the $R_{K}$ anomaly with texture $A_{1}$ [46]. Other viable two texture-zeros are systematically realised in Ref. [47] by considering the $U(1)_{B-2 L_{\alpha}-L_{\beta}}$ gauge symmetry with three right-handed singlets. In this paper, we provide another viable approach. Under the same flavor dependent $U(1)_{B-2 L_{\alpha}-L_{\beta}}$ gauge symmetry, we introduce only two right-handed singlets but two inert scalars, leading to different texture-zeros compared with Ref. [47]. In aspect of predicted phenomenology, the texture $B_{1}$ con- 
sidered in Ref. [47] is marginally allowed by current Planck result for $\sum m_{i}<0.12 \mathrm{eV}$ [48], we thus consider texture $A_{1}$ with latest neutrino oscillation data [49] as the benchmark model. In this case, the gauge symmetry is $U(1)_{B-2 L_{e}-L_{\tau}}$ in our approach.

The rest of this paper is organised as follows. Start with classic scotogenic model in Sect. 2, we first discuss the realization of texture-zeros in scotogenic model with $U(1)_{B-2 L_{\alpha}-L_{\beta}}$ gauge symmetry in a general approach. Then

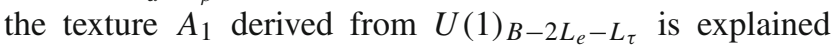
in detail. The corresponding phenomenological predictions, such as neutrino mixing parameters, lepton flavor violation rate, dark matter and highlights of collider signatures are presented in Sect. 3. Finally, conclusions are summarised in Sect. 4

\section{The model setup}

\subsection{Classic scotogenic model}

In the classic scotogenic model proposed by $\mathrm{Ma}$ [1], three right-handed fermion singlets $N_{R i}(i=1 \sim 3)$ and an inert scalar doublet field $\eta=\left(\eta^{+}, \eta^{0}\right)$ are added to the SM. In addition, a discrete $Z_{2}$ symmetry is imposed for the new fields in order to forbid the tree-level neutrino Yukawa interaction and stabilize the DM candidate. The relevant interactions for neutrino masses generation are given by

$\mathcal{L} \supset h_{\alpha i} \bar{L}_{\alpha} \tilde{\eta} N_{R i}+\frac{1}{2} M_{N} \bar{N}_{R}^{c} N_{R}+\frac{1}{2} \lambda\left(\Phi^{\dagger} \eta\right)^{2}+$ h.c..

The mass matrix $M_{N}$ can be diagonalized by an unitary matrix $V$ satisfying

$V^{T} M_{N} V=\hat{M}_{N} \equiv \operatorname{diag}\left(M_{N 1}, M_{N 2}, M_{N 3}\right)$.

Due to the $Z_{2}$ symmetry, the neutrino masses are generated at one-loop level, as show in left pattern of Fig. 1. The neutrino mass matrix can be computed exactly, i.e.



Fig. 1 Radiative neutrino mass at one-loop. Left pattern is for classic scotogenic model, while right pattern is the additional contribution in our models

$$
\begin{aligned}
\left(M_{\nu}\right)_{\alpha \beta}= & \frac{1}{32 \pi^{2}} \sum_{k} h_{\alpha i} V_{i k} h_{\beta j} V_{j k} M_{N k} \\
& \times\left[\frac{m_{R}^{2}}{m_{R}^{2}-M_{N k}^{2}} \log \left(\frac{m_{R}^{2}}{M_{N k}^{2}}\right)-\frac{m_{I}^{2}}{m_{I}^{2}-M_{N k}^{2}} \log \left(\frac{m_{I}^{2}}{M_{N k}^{2}}\right)\right]
\end{aligned}
$$

where $m_{R}$ and $m_{I}$ are the masses of $\sqrt{2} \Re \eta^{0}$ and $\sqrt{2} \Im \eta^{0}$. If we assume $m_{0}^{2} \equiv\left(m_{R}^{2}+m_{I}^{2}\right) / 2 \gg M_{N k}^{2}, M_{v}$ are then given by

$$
\begin{aligned}
\left(M_{\nu}\right)_{\alpha \beta} & \simeq-\frac{1}{32 \pi^{2}} \frac{\lambda v^{2}}{m_{0}^{2}} \sum_{k} h_{\alpha i} V_{i k} h_{\beta j} V_{j k} M_{N k} \\
& =-\frac{1}{32 \pi^{2}} \frac{\lambda v^{2}}{m_{0}^{2}}\left(h M_{N} h^{T}\right)_{\alpha \beta}
\end{aligned}
$$

The neutrino mass matrix $M_{v}$ is diagonalized as

$U_{\mathrm{PMNS}}^{T} M_{v} U_{\mathrm{PMNS}}=\hat{m}_{v} \equiv \operatorname{diag}\left(m_{1}, m_{2}, m_{3}\right)$,

where $U_{\mathrm{PMNS}}$ is the neutrino mixing matrix denoted as

$$
\begin{aligned}
U_{\mathrm{PMNS}}= & \left(\begin{array}{lll}
c_{12} c_{13} & s_{12} c_{13} & s_{13} \\
-c_{12} s_{23} s_{13}-s_{12} c_{23} e^{-i \delta} & -s_{12} s_{23} s_{13}+c_{12} c_{23} e^{-i \delta} & s_{23} c_{13} \\
-c_{12} c_{23} s_{13}+s_{12} s_{23} e^{-i \delta} & -s_{12} c_{23} s_{13}-c_{12} s_{23} e^{-i \delta} & c_{23} c_{13}
\end{array}\right) \\
& \times \operatorname{diag}\left(e^{i \rho}, e^{i \sigma}, 1\right)
\end{aligned}
$$

Here, we define $c_{i j}=\cos \theta_{i j}$ and $s_{i j}=\sin \theta_{i j}(i j=$ $12,23,13)$ for short, $\delta$ is the Dirac phase and $\rho, \sigma$ are the two Majorana phases as in Ref. [34].

\subsection{Two texture-zeros in scotogenic model}

In this section, we demonstrate a class of scotogenic models with $G_{S M} \times U(1)_{B-2 L_{\alpha}-L_{\beta}} \times Z_{2}$ gauge symmetry where two texture-zero structures in $M_{v}$ are successfully realized. The particle content and corresponding charge assignments are listed in Table 1. In the fermion sector, we introduce two right-handed $S U(2)_{L}$ singlets $N_{R 1}$ and $N_{R 2}$ and assume they carry the same no-zero $B-2 L_{\alpha}-L_{\beta}$ charges as two of SM leptons respectively. Noticeably, if one further introduce one additional $N_{R 3}$ with zero $B-2 L_{\alpha}-L_{\beta}$ charge, the approach considered in Ref. [47] are then reproduced. In terms of gauged $U(1)_{B-2 L_{\alpha}-L_{\beta}}$ symmetry, the anomaly free conditions should be considered first and we find all anomalies are zero because

$$
\begin{aligned}
& {\left[S U(3)_{C}\right]^{2} U(1)_{X}: 3 \times \frac{1}{2}\left(\frac{2}{3}-\frac{1}{3}-\frac{1}{3}\right)=0} \\
& U(1)_{Y}\left[U(1)_{X}\right]^{2}: 3\left[6\left(\frac{1}{6}\right)-3\left(\frac{2}{3}\right)-3\left(\frac{1}{3}\right)\right]\left(\frac{1}{3}\right)^{2} \\
& \quad+\left[2\left(-\frac{1}{2}\right)-(-1)\right]\left[(-1)^{2}+(-2)^{2}\right]=0 \\
& {\left[S U(2)_{L}\right]^{2} U(1)_{X}: \frac{1}{2}\left[3 \times 3\left(\frac{1}{3}\right)+(-1)+(-2)\right]=0}
\end{aligned}
$$


Table 1 Particle content and corresponding charge assignments

\begin{tabular}{|c|c|c|c|c|c|c|c|c|c|c|c|c|c|}
\hline \multirow[t]{2}{*}{ Group } & \multicolumn{8}{|c|}{ Lepton fields } & \multicolumn{5}{|c|}{ Scalar fields } \\
\hline & $L_{\alpha}$ & $\ell_{\alpha R}$ & $L_{\beta}$ & $\ell_{\beta R}$ & $L_{\gamma}$ & $\ell_{\gamma R}$ & $N_{R 1}$ & $N_{R 2}$ & $\Phi$ & $\eta_{1}$ & $\eta_{2}$ & $S_{1}$ & $S_{2}$ \\
\hline$S U(2)_{L}$ & 2 & 1 & 2 & 1 & 2 & 1 & 0 & 0 & 2 & 2 & 2 & 1 & 1 \\
\hline$U(1)_{Y}$ & $-\frac{1}{2}$ & -1 & $-\frac{1}{2}$ & -1 & $-\frac{1}{2}$ & -1 & 1 & 1 & $\frac{1}{2}$ & $\frac{1}{2}$ & $\frac{1}{2}$ & 0 & 0 \\
\hline$Z_{2}$ & + & + & + & + & + & + & - & - & + & - & - & + & + \\
\hline$U(1)_{B-2 L_{\alpha}-L_{\beta}}$ & -2 & -2 & -1 & -1 & 0 & 0 & -1 & -2 & 0 & -1 & 0 & 2 & 3 \\
\hline
\end{tabular}

$$
\begin{aligned}
& {\left[U(1)_{Y}\right]^{2} U(1)_{X}: 3\left[6\left(\frac{1}{6}\right)^{2}-3\left(\frac{2}{3}\right)^{2}-3\left(\frac{1}{3}\right)^{2}\right]\left(\frac{1}{3}\right)} \\
& +\left[2\left(-\frac{1}{2}\right)^{2}-(-1)^{2}\right](-1-2)=0 \\
& U(1)_{X}^{3}: 2(-1)^{3}-2(-1)^{3}+2(-2)^{3}-2(-2)^{3}=0 \\
& {[\text { Gravity }]^{2} U(1)_{X}: 2(-1)-2(-1)+2(-2)-2(-2)=0}
\end{aligned}
$$

Let us now discuss the scotogenic realizations of two texturezeros in $M_{\nu}$. With two $N_{R}$ components, $h$ and $M_{N}$ are $3 \times 2$ and $2 \times 2$ matrices respectively. From Eq. (4), it is clear that the texture-zeros of $M_{v}$ can be attributed to the texture-zeros in $h$ and $M_{N}$ matrices. In the original scotogenic model with an inert scalar doublet $\eta$ and two $N_{R}$ fields, the charge assignments for $U(1)_{B-2 L_{\alpha}-L_{\beta}}$ gauge symmetry give rise to only two Yukawa terms for $h_{\alpha i} \bar{L}_{\alpha} \tilde{\eta} N_{R i}(\alpha=e, \mu, \tau, i=1,2)$. In this case, at least two texture-zeros are placed in the same line of $h$ matrix, being therefore excluded experimentally. In order to accommodate the realistic neutrino mixing data, the scotogenic model are extended where, in scalar sector, two inert doublet $\eta_{1}$ and $\eta_{2}$ are introduced (see Table 1). In addition, two scalar singlet $S_{1}$ and $S_{2}$ are added so that $U(1)_{B-2 L_{\alpha}-L_{\beta}}$ symmetry is spontaneously breaking after $S_{1,2}$ get the vacuum expectation value (VEV) $\left\langle S_{1,2}\right\rangle=v_{1,2} / \sqrt{2}$. Note that $N_{R i}$ and $\eta_{i}$ are odd under the discrete $Z_{2}$ symmetry. Since we have two inert scalars, the relevant scalar interactions for the loop-induced neutrino masses is given by

$\mathcal{L}_{S} \supset \frac{\lambda}{\Lambda}\left(\Phi^{\dagger} \eta_{1}\right)^{2} S_{1}+\lambda^{\prime}\left(\Phi^{\dagger} \eta_{2}\right)^{2}+\left(\lambda_{1}\left(\eta_{2}^{\dagger} \eta_{1}\right) S_{1}^{*} S_{2}+\right.$ h.c. $)$,

where $\Lambda$ is a new high energy scale and the first term is a dimension-five operator guaranteed by the accidental $U(1)_{B-2 L_{\alpha}-L_{\beta}}$ symmetry. One can achieve the effective operator by simply adding a new scalar singlet $\rho \sim$ $(1,0,1,-)$ so that in scalar sector $\mathcal{L}_{S} \supset \mu\left(\Phi^{\dagger} \eta_{1}\right) \rho^{\dagger}+$ $\mu^{\prime} \rho^{2} S_{1}$ is allowed. Then the effective interaction $\lambda\left(\Phi^{\dagger} \eta_{1}\right)^{2} S_{1} / \Lambda$ is obtained by integrate the $\rho$ field out of $\mathcal{L}_{S}$ sector. In the following analysis, we adopt the expression of effective operator in Eq. (8) and do not consider its specific realization in detail. After $S_{1,2}$ develop VEVs, the $\lambda_{1}$-term will induce mixing between $\eta_{1}$ and $\eta_{2}$. In following studies, we set $\lambda_{1}=0$, thus no mixing between $\eta_{1}$ and $\eta_{2}$ for simplicity.

The neutrinos acquire their tiny masses radiatively though the one-loop diagrams depicted in Fig. 1. As a case study, we consider the $U(1)_{B-2 L_{e}-L_{\tau}}$ gauge symmetry, under which the flavor dependent Yukawa interactions are given by

$$
\begin{aligned}
-\mathcal{L}_{\mathrm{Y}}= & h_{\mu 1} \bar{L}_{\mu} \tilde{\eta}_{1} N_{R 1}+h_{\tau 2} \bar{L}_{\tau} \tilde{\eta}_{1} N_{R 2} \\
& +f_{\tau 1} \bar{L}_{\tau} \tilde{\eta}_{2} N_{R 1}+f_{e 2} \bar{L}_{e} \tilde{\eta}_{2} N_{R 2} \\
& +y_{11} \overline{N_{R 1}^{c}} N_{R 1} S_{1}+y_{12}\left(\overline{N_{R 1}^{c}} N_{R 2}\right. \\
& \left.+\overline{N_{R 2}^{c}} N_{R 1}\right) S_{2}+\text { h.c.. }
\end{aligned}
$$

From the charge assignment, the texture of above Yukawa couplings are

$h=\left(\begin{array}{ll}0 & 0 \\ h_{\mu 1} & 0 \\ 0 & h_{\tau 2}\end{array}\right), \quad f=\left(\begin{array}{ll}0 & f_{e 2} \\ 0 & 0 \\ f_{\tau 1} & 0\end{array}\right), \quad y=\left(\begin{array}{ll}y_{11} & y_{12} \\ y_{12} & 0\end{array}\right)$.

Therefore, the neutrino mass matrix is formulated by two different contribution coming from $\eta_{1}$ and $\eta_{2}$, namely,

$\left(M_{v}\right) \propto h M_{N} h^{T}+f M_{N} f^{T}$,

with further assumption $\Lambda=\left\langle S_{1}\right\rangle$ and $\lambda / m_{\eta_{1}}^{2}=\lambda^{\prime} / m_{\eta_{2}}^{2}$. Provided all the element in $M_{N}$ to be equal, then from the texture structure in Eq. (10) and using Eq. (11) we have the $M_{\nu}$ as

$M_{\nu} \propto\left(\begin{array}{lll}0 & 0 & f_{e 2} f_{\tau 1} \\ 0 & h_{\mu 1}^{2} & h_{\mu 1} h_{\tau 2} \\ f_{e 2} f_{\tau 1} & h_{\mu 1} h_{\tau 2} & f_{\tau 1}^{2}\end{array}\right)$

which is texture $A_{1}$ allowed by experimental data [34, 35]. Other possible realizations of two-zero textures with $U(1)_{B-2 L_{\alpha}-L_{\beta}}$ can then be easily obtained in a similar approach. In Table 2, we summarize all the six textures realised by $U(1)_{B-2 L_{\alpha}-L_{\beta}}$ in our approach. According to Ref. [35], texture $A_{1}$ and $A_{2}$ predict $\sum m_{i} \sim 0.07 \mathrm{eV}$, hence they are allowed by Planck limit $\sum m_{i}<0.12 \mathrm{eV}$ [48]. Texture $B_{3}$ and $B_{4}$ predict $\sum m_{i} \gtrsim 0.15 \mathrm{eV}$, thus they are marginally allowed if certain mechanism is introduced to modify cosmology data. Texture $D_{1}$ and $D_{2}$ are 
Table 2 Two texture-zeros and corresponding $U(1)_{B-2 L_{\alpha}-L_{\beta}}$ symmetry. Here, $\times$ denotes a nonzero matrix element

\begin{tabular}{llcc}
\hline Texture of $M_{v}$ & Group & Texture of $M_{v}$ & Group \\
\hline$A_{1}:\left(\begin{array}{ccc}0 & 0 & \times \\
0 & \times & \times \\
\times & \times & \times\end{array}\right)$ & $U(1)_{B-2 L_{e}-L_{\tau}}$ & $A_{2}:\left(\begin{array}{ccc}0 & \times & 0 \\
\times & \times & \times \\
0 & \times & \times\end{array}\right)$ & $U(1)_{B-2 L_{e}-L_{\mu}}$ \\
$B_{3}:\left(\begin{array}{ccc}\times & 0 & \times \\
0 & 0 & \times \\
\times & \times & \times\end{array}\right)$ & $B_{4}:\left(\begin{array}{ccc}\times & \times & 0 \\
\times & \times & \times \\
0 & \times & 0\end{array}\right)$ & $U(1)_{B-2 L_{\tau}-L_{\mu}}$ & Allowed \\
$D_{1}:\left(\begin{array}{ccc}\times & \times & \times \\
\times & 0 & 0 \\
\times & 0 & \times\end{array}\right)$ & $U(1)_{B-2 L_{\mu}-L_{\tau}}$ & $U(1)_{B-2 L_{\tau}-L_{e}}$ & Excluded \\
\hline
\end{tabular}

Table 3 Same as 2, but for one texture-zero

\begin{tabular}{|c|c|c|c|c|c|}
\hline Texture of $M_{v}$ & Group & Texture of $M_{v}$ & Group & Status texture of $M_{v}$ & Group \\
\hline$\left(\begin{array}{lll}0 & \times & \times \\
\times & \times & \times \\
\times & \times & \times\end{array}\right)$ & $U(1)_{B-2 L_{e}-L_{\mu, \tau}}$ & $\left(\begin{array}{ccc}\times & \times & \times \\
\times & 0 & \times \\
\times & \times & \times\end{array}\right)$ & $U(1)_{B-2 L_{\mu}-L_{e, \tau}}$ & $\left(\begin{array}{ccc}\times & \times & \times \\
\times & \times & \times \\
\times & \times & 0\end{array}\right)$ & $U(1)_{B-2 L_{\tau}-L_{e, \mu}}$ \\
\hline
\end{tabular}

already excluded by neutrino oscillation data. The following phenomenological predictions are based on texture $A_{1}$ with

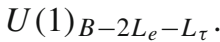

Note if $\lambda_{1} \neq 0$, hence mixing between $\eta_{1}$ and $\eta_{2}$ exists, then new contributions as $h M_{N} f^{T}+f M_{N} h^{T}$ to neutrino masses are possible. In this way, the resulting neutrino mass matrix will only have one texture-zero [50], thus less constrained and less predictive than the two texture-zero. The obtained one texture-zeros are presented in Table 3, which are all allowed by neutrino oscillation data [50]. Since two different gauge symmetry lead to same one texture-zero, it is then not possible to distinguish them only by precise neutrino oscillation measurements.

In the mass eigenstate of heavy Majorana fermion $N_{i}$, the corresponding Yukawa couplings with leptons are easily obtained by

$$
\begin{aligned}
& h^{\prime}=h V=\left(\begin{array}{ll}
0 & 0 \\
h_{\mu 1} V_{11} & h_{\mu 1} V_{12} \\
h_{\tau 2} V_{21} & h_{\tau 2} V_{22}
\end{array}\right), \\
& f^{\prime}=f V=\left(\begin{array}{ll}
f_{e 2} V_{21} & f_{e 2} V_{22} \\
0 & 0 \\
f_{\tau 1} V_{11} & f_{\tau 1} V_{12}
\end{array}\right) .
\end{aligned}
$$

For the $Z_{2}$-even scalars, the CP-even scalars in weak-basis $\left(\sqrt{2} \Re \Phi^{0}, \sqrt{2} \Re S_{1}, \sqrt{2} \Re S_{2}\right)$ mix into mass-basis $\left(h, H_{1}, H_{2}\right)$ with mass spectrum $M_{h} \sim M_{H_{1}}<M_{H_{2}}$. Without loss of generality, we further assume the mixing angle between ( $h, H_{1}$ ) to be $\alpha$ and vanishing mixing angles between $H_{2}$ and $h / H_{1}$ for simplicity. Mass of $H_{2}$ is fixed to be $m_{H_{2}}=10$ TeV. The would-be Goldstone boson $\Phi^{+}, \sqrt{2} \Im \Phi^{0}, \sqrt{2} \Im S_{2}$ are absorbed by gauge boson $W^{+}, Z, Z^{\prime}$ respectively, leav- ing $\sqrt{2} \Im S_{1}$ a pseudo-Goldstone, e.g., Majoron $J$. This Majoron can be assigned to the spontaneous breaking of the global $U(1)_{L}$ lepton number symmetry, under which $L, \ell, N_{R} \sim 1, S_{1,2} \sim-2$. All the renormalizable interactions preserve this global symmetry, but the effective interaction $\lambda\left(\Phi^{\dagger} \eta_{1}\right)^{2} S_{1} / \Lambda$ clearly breaks this $U(1)_{L}$ symmetry. This term will induce an effective mass term for the Majoron at one loop level, with $m_{J} \sim \lambda v^{2} /(4 \pi \Lambda)$. Typically, for $\Lambda \sim 10 \mathrm{TeV}$ and $\lambda \sim 10^{-10}$ to acquire correct neutrino masses [51], we have $m_{J} \sim 10^{-2} \mathrm{eV}$. Due to the charge assignment, the Majoron here does not mix with SM Goldstone bosons, thus it is a pure singlet under SM gauge group. From Eq. (9), we know that this Majoron only directly couples to the inert $N_{R}$, and the Yukawa coupling $\bar{L} \tilde{\eta} N_{R}$ could mediate Majoron coupling to leptons at one loop level. For $10^{-2} \mathrm{eV}$ scale Majoron $J$, it can only decay into light neutrinos. Since texture of $M_{v}$ in Eq. (4) is derived by $m_{0}^{2} \gg M_{N k}^{2}$, only fermion DM is allowed in this paper.

\section{Phenomenology}

\subsection{Neutrino mixing}

As shown in the previous section, the flavor dependent

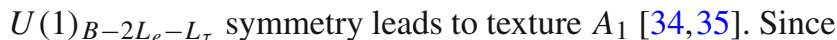
$\left(M_{\nu}\right)_{e e}=0$, the predicted effective Majorana neutrino mass $\langle m\rangle_{e e}$ is exactly zero for the neutrinoless double-beta decay. Therefore, only normal hierarchy is allowed in this model [52,53]. Following the procedure in Ref. [34], we now update the predictions of neutrino oscillation data with latest global analysis results [49]. 

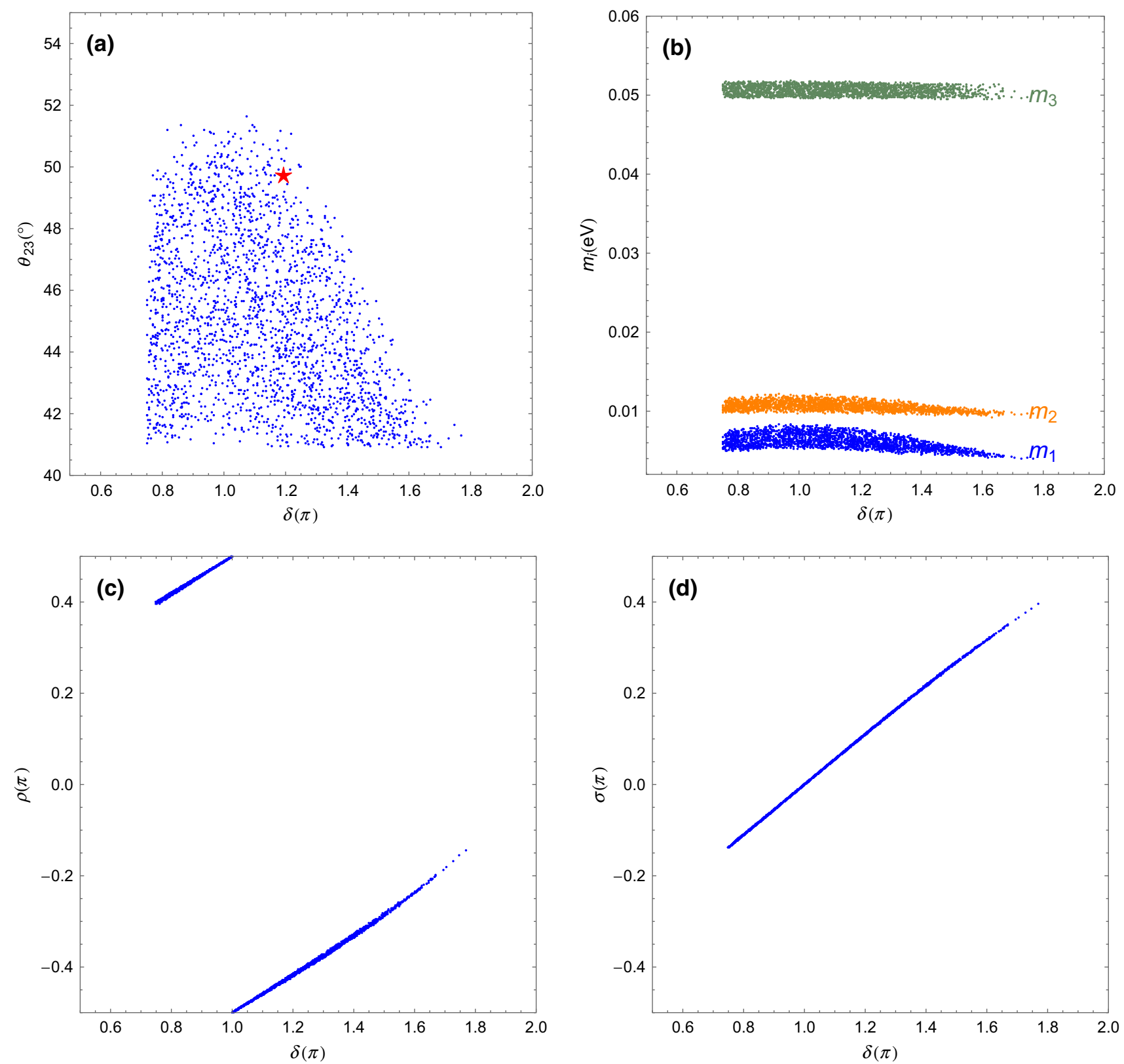

Fig. 2 Allowed samples of $A_{1}$ texture with neutrino oscillation data varied in $3 \sigma$ range of Ref. [49]. In pattern $\mathbf{a}$, the red star $\star$ stands for the best fit point from global analysis

In Fig. 2, we show the scanning results of texture $A_{1}$. It is worth to note that the best fit value of neutrino oscillation parameters by global analysis [49] is only marginally consistent with predictions of texture $A_{1}$, which is clearly seen in Fig. 2a. From Fig. 2b, we obtain that $m_{1} \sim 0.007 \mathrm{eV}$, $m_{2} \sim 0.01 \mathrm{eV}$, and $m_{3} \approx \sqrt{\Delta m^{2}} \sim 0.05 \mathrm{eV}$. The resulting sum of neutrino mass is then $\sum m_{i} \sim 0.07 \mathrm{eV}$, thus it satisfies the bound from cosmology, i.e., $\sum m_{i}<0.12 \mathrm{eV}[48,54]$. The Dirac phase should fall in the range $\delta \in[0.75 \pi, 1.77 \pi]$, meanwhile Fig. $2 \mathrm{c}$, d indicate that $\rho \approx \frac{\delta}{2}$ and $\sigma \approx \frac{\delta}{2}-\frac{\pi}{2}$.

Instead of the marginally best fit neutrino oscillation parameters, we take $\delta=\pi$ and $\theta_{23}=46^{\circ}$ with other oscil- lation parameters being the best fit value in Ref. [49] as the benchmark point for illustration, which leads to the following neutrino mass structure

$$
M_{v}=\left(\begin{array}{lll}
0 & 0 & 0.0110 \\
0 & 0.0293 & 0.0219 \\
0.0110 & 0.0219 & 0.0256
\end{array}\right) \mathrm{eV}
$$

By comparing the analytic $M_{v}$ in Eq. (12) and the numerical $M_{v}$ in Eq. (14), one can easily reproduce the observed neutrino oscillation data by requiring 
$\begin{aligned} \frac{h_{\tau 2}}{h_{\mu 1}}: \frac{f_{\tau 1}}{h_{\mu 1}}: \frac{f_{e 2}}{h_{\mu 1}} & =\frac{\left(M_{\nu}\right)_{\mu \tau}}{\left(M_{\nu}\right)_{\mu \mu}}: \sqrt{\frac{\left(M_{\nu}\right)_{\tau \tau}}{\left(M_{\nu}\right)_{\mu \mu}}}: \frac{\left(M_{\nu}\right)_{e \tau}}{\sqrt{\left(M_{\nu}\right)_{\mu \mu}\left(M_{\nu}\right)_{\tau \tau}}} \\ & \simeq 0.745: 0.933 \cdot 0.401\end{aligned}$

Hence, we can take $h_{\mu 1}$ as free parameters and determine the other three Yukawa coupling by using above ratios. The overall neutrino mass scale is then determined by $\lambda v^{2} M_{N} h_{\mu 1}^{2} /\left(32 \pi^{2} m_{0}^{2}\right) \approx 0.0293 \mathrm{eV}$.

\subsection{Lepton flavor violation}

The new Yukawa interactions of the form $\bar{L} \tilde{\eta} N_{R}$ will contribute to lepton flavor violation (LFV) processes $[55,56]$. In this work, we take the radiative decay $\ell_{i} \rightarrow \ell_{j} \gamma$ for illustration. Generally, the Yukawa couplings are tightly constrained


vanishing mixing angle $\theta$, it is clear from Eq. (9) that $\eta_{1}^{ \pm}\left(\eta_{2}^{ \pm}\right)$ will only induce $\tau \rightarrow \mu \gamma(\tau \rightarrow e \gamma)$ at one-loop level. It is worth to note that the most stringent $\mu \rightarrow e \gamma$ decay is missing at one-loop level. Hence, if the ongoing experiments observe $\tau \rightarrow \mu(e) \gamma$ but no $\mu \rightarrow e \gamma$, this model will be favored. The corresponding branching ratios are calculated as

$$
\begin{aligned}
& \operatorname{BR}(\tau \rightarrow \mu \gamma) \\
& =\frac{3 \alpha}{64 \pi G_{F}^{2}}\left|\sum_{i=1}^{2} \frac{\left(h_{\mu 1} V_{1 i}\right)\left(h_{\tau 2} V_{2 i}\right)^{*}}{M_{\eta_{1}}^{2}} F\left(\frac{M_{N i}^{2}}{M_{\eta_{1}}^{2}}\right)\right|^{2} \operatorname{BR}\left(\tau \rightarrow \mu \nu_{\tau} \bar{\nu}_{\mu}\right), \\
& \operatorname{BR}(\tau \rightarrow e \gamma) \\
& =\frac{3 \alpha}{64 \pi G_{F}^{2}}\left|\sum_{i=1}^{2} \frac{\left(f_{e 2} V_{2 i}\right)\left(f_{\tau 1} V_{1 i}\right)^{*}}{M_{\eta_{2}}^{2}} F\left(\frac{M_{N i}^{2}}{M_{\eta_{2}}^{2}}\right)\right|^{2} \operatorname{BR}\left(\tau \rightarrow e v_{\tau} \bar{\nu}_{e}\right),
\end{aligned}
$$

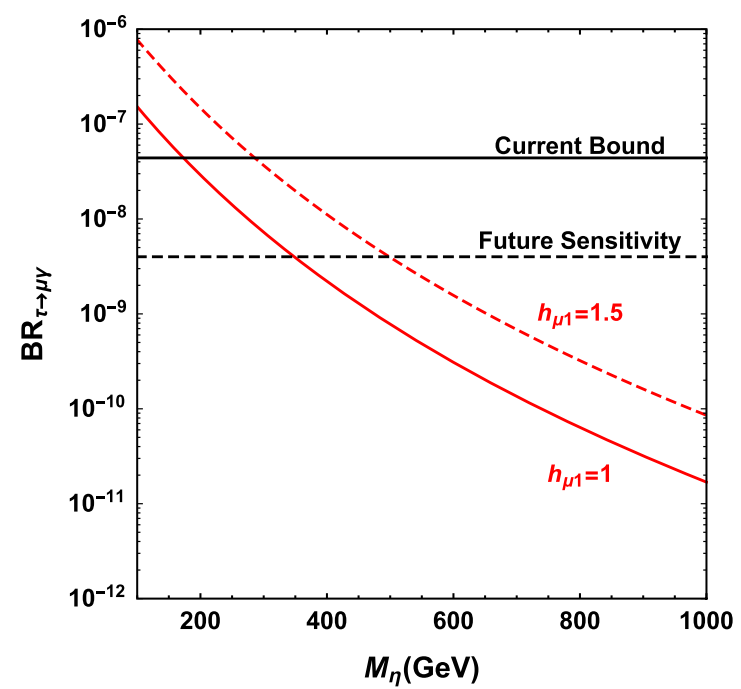

where the loop function $F(x)$ is

$$
F(x)=\frac{1-6 x+3 x^{2}+2 x^{3}-6 x^{2} \ln x}{6(1-x)^{4}} .
$$

In the limit of degenerate $M_{N}$, we have

$\operatorname{BR}(\tau \rightarrow \ell \gamma) \propto\left|\sum_{i=1}^{2} V_{1 i} V_{2 i}^{*}\right|^{2}=\left|\left(V V^{\dagger}\right)_{12}\right|^{2}=0$,

where in the last step, we have considered the fact that $V$ is an unitary matrix. Therefore, cancellations between the contributions of two $N_{i}$ are also expected even in the case of nearly degenerate $M_{N}$. In Fig. 3, we show the predictions for $\tau \rightarrow \mu \gamma$ and $\tau \rightarrow e \gamma$. Although constraint on $\operatorname{BR}(\tau \rightarrow e \gamma)$ is slightly more stringent than $\operatorname{BR}(\tau \rightarrow \mu \gamma)$, the predicted $\mathrm{BR}(\tau \rightarrow e \gamma)$ is much smaller than $\mathrm{BR}(\tau \rightarrow \mu \gamma)$. It is clear that the current bound is quite loose, e.g., $M_{\eta} \gtrsim 200 \mathrm{GeV}$ with $h_{\mu 1}=1$ can be allowed.

Although the Yukawa interaction $\bar{L}_{\mu} \tilde{\eta}_{1} N_{i}$ can not induce $\mu \rightarrow e \gamma$ at one-loop, it does contribute to muon anomalous magnetic moment [59]

$\Delta a_{\mu}=-\sum_{i=1}^{2} \frac{\left|h_{\mu 1} V_{1 i}\right|^{2} M_{\mu}^{2}}{16 \pi^{2} M_{\eta_{1}}^{2}} F\left(\frac{M_{N i}^{2}}{M_{\eta_{1}}^{2}}\right)$.

Comparing with $\operatorname{BR}(\tau \rightarrow \ell \gamma)$, there is no cancellations between the contributions of two $N_{i}$. However, the total contribution to $\Delta a_{\mu}$ is negative, while the observed discrepancy $\Delta a_{\mu}=a_{\mu}^{\mathrm{EXP}}-a_{\mu}^{\mathrm{SM}}=(261 \pm 78) \times 10^{-11}$ is positive [60]. Thus, the Yukawa interaction $\bar{L}_{\mu} \tilde{\eta}_{1} N_{R 1}$ can not explain the $(g-2)_{\mu}$ anomaly, and some other new physics is required [59]. On the other hand, since a too large negative contribution to $\Delta a_{\mu}$ is not favored, we consider a theoretical limit, i.e., $\left|\Delta a_{\mu}\right|<10^{-10}$ in the following. The results are shown



Fig. 3 Predictions for $\tau \rightarrow \mu \gamma$ (left) and $\tau \rightarrow e \gamma$ (right) with corresponding current bound [57] and future sensitivity [58]. In these figures, we have fixed $M_{N_{1}}=200 \mathrm{GeV}$ 


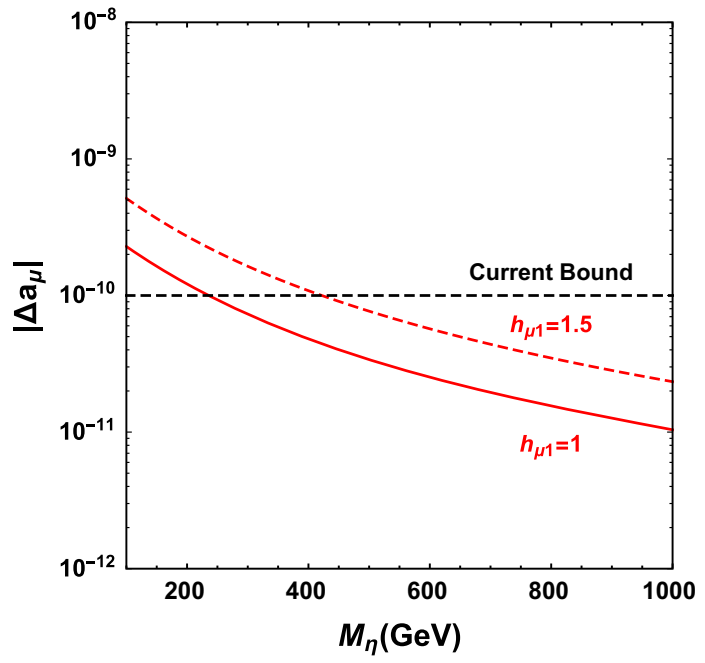

Fig. 4 Predictions for $\left|\Delta a_{\mu}\right|$. In this figures, we have fix $M_{N_{1}}=200$ $\mathrm{GeV}$

in Fig. 4. We find that the bound from $\left|\Delta a_{\mu}\right|<10^{-10}$ is actually slightly more stringent than $\operatorname{BR}(\tau \rightarrow \mu \gamma)$.

\subsection{Dark matter}

In this work, $N_{1}$ is the viable DM candidate. In the original scotogenic model [1], the possible annihilation channels are $N_{1} N_{1} \rightarrow \ell^{+} \ell^{-}, \bar{v} v$ via the Yukawa interaction $\bar{L}_{\ell} \tilde{\eta} N_{1}$ [61]. However, such annihilation channels are tightly constrained by non-observation of LFV [51]. Thanks to relative loose constraints from $\tau$ decays, the scanning results of Ref. [51] suggested that $N_{1}$ should have a large coupling to $L_{\tau}$. Thus, the dominant annihilation channel is $\tau^{+} \tau^{-}$and $\bar{\nu}_{\tau} v_{\tau}$ with $M_{N_{1}} \lesssim 3 \mathrm{TeV}$.

Quite different from the original scotogenic model [1], the LFV process is either vanishing or suppressed in this flavor dependent model. As shown in Sect. 3.2, $\mathcal{O}$ (1) Yukawa couplings are allowed by current experimental bounds. In the following quantitative investigation, we consider a special scenario, i.e., $M_{\eta_{1}}=M_{\eta_{2}}=M_{\eta}$ for simplicity. In the limit of vanishing lepton masses, the Yukawa-portal annihilation cross section is $[61,62]$

$$
\begin{aligned}
\sigma v_{\text {rel }} & =a+b v_{\text {rel }}^{2} \\
& =0+\sum_{\alpha, \beta}\left|h_{\alpha 1}^{\prime} h_{\beta 1}^{*}+f_{\alpha 1}^{\prime} f_{\beta 1}^{\prime *}\right|^{2} \frac{r^{2}\left(1-2 r+2 r^{2}\right)}{24 \pi M_{N_{1}}^{2}} v_{\text {rel }}^{2},
\end{aligned}
$$

where $v_{\text {rel }}$ is the relative speed, $h^{\prime}$ and $f^{\prime}$ are defined in Eq. (13), $r=M_{N_{1}}^{2} /\left(M_{\eta}^{2}+M_{N_{1}}^{2}\right)$. The thermally averaged cross section is calculated as $\left\langle\sigma v_{\text {rel }}\right\rangle=a+6 b / x_{f}$, where the freeze-out parameter $x_{f}=M_{N_{1}} / T_{f}$ is obtained by numerically solving



Fig. 5 Predicted relic density as a function of $m_{\eta}$, where we have fix $M_{N_{1}}=200 \mathrm{GeV}$. The green line corresponds to the observed relic density $\Omega h^{2}=0.120 \pm 0.001[48]$

$x_{f}=\ln \left(\frac{0.038 M_{\mathrm{Pl}} M_{N_{1}}\left\langle\sigma v_{\mathrm{rel}}\right\rangle}{\sqrt{g_{*} x_{f}}}\right)$.

The relic density is then calculated as [63]

$\Omega h^{2}=\frac{1.07 \times 10^{9} \mathrm{GeV}^{-1}}{M_{\mathrm{Pl}}} \frac{x_{f}}{\sqrt{g_{*}}} \frac{1}{a+3 b / x_{f}}$,

where $M_{\mathrm{Pl}}=1.22 \times 10^{19} \mathrm{GeV}$ is the Planck mass, $g_{*}$ is the number of relativistic degrees of freedom. The numerical results are depicted in Fig. 5. Provided the mass of the DM candidate is $M_{N_{1}}=200 \mathrm{GeV}$, then the observed relic density is interpreted by $h_{\mu 1}=1, M_{\eta}=366 \mathrm{GeV}$ or $h_{\mu 1}=1.5, M_{\eta}=640 \mathrm{GeV}$. That is to say, $h_{\mu 1} \sim \mathcal{O}(1)$ is required to obtain correct relic density, and the larger $h_{\mu 1}$ is, the larger the mass splitting $M_{\eta}-M_{N_{1}}$ is.

In addition to the Yukawa-portal interaction, $N_{1}$ can also annihilate via the Higgs-portal and $Z^{\prime}$-portal interactions [64-73]. In these two scenarios, $M_{N_{1}} \simeq M_{h, H} / 2$ or $\simeq M_{Z^{\prime}} / 2$ are usually required to obtain correct relic density [74]. If the additional scalar singlet scalar $H$ is lighter than $N_{1}$, then the annihilation channel $N_{1} N_{1} \rightarrow H H$ with $H \rightarrow b \bar{b}$ is able to explain the Fermi-LAT gamma-ray excess at the Galactic center $[75,76]$.

The spin-independent DM-nucleon scattering cross section is dominantly mediated by scalar interactions, which is given by

$\sigma^{\mathrm{SI}}=\frac{4}{\pi}\left(\frac{M_{p} M_{N_{1}}}{M_{p}+M_{N_{1}}}\right)^{2} f_{p}^{2}$,

where $M_{p}$ is the proton mass and the hadronic matrix element $f_{p}$ reads 


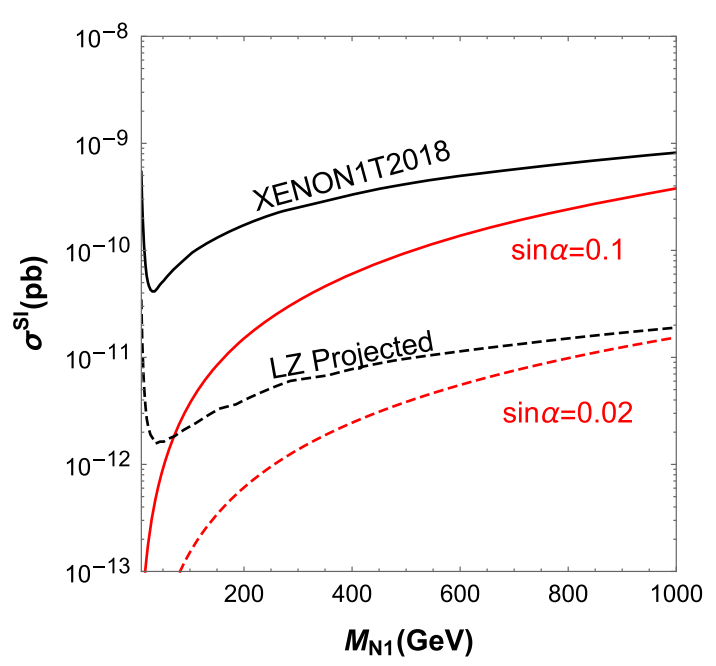

Fig. 6 Spin-independent cross section as a function of $M_{N_{1}}$. The black solid and dashed line correspond to current XENON1T [78] and future LZ [79] limits, respectively. In this figure, we have set $M_{H_{1}}=500 \mathrm{GeV}$ and $v_{S}=10 \mathrm{TeV}$

$$
\begin{aligned}
\frac{f_{p}}{M_{p}}= & \sum_{q=u, d, s} f_{T q}^{p} \frac{\alpha_{q}}{M_{q}} \\
& +\frac{2}{27}\left(1-\sum_{q=u, d, s} f_{T q}^{p}\right) \sum_{q=c, b, t} \frac{\alpha_{q}}{M_{q}} .
\end{aligned}
$$

and the effective vertex

$\frac{\alpha_{q}}{M_{q}}=-\frac{y_{N_{1}}}{\sqrt{2} v} \sin 2 \alpha\left(\frac{1}{M_{h}^{2}}-\frac{1}{M_{H_{1}}^{2}}\right)$,

Here, $y_{N_{1}}=y_{11} V_{11}^{2}$ is the effective Yukawa coupling of $N_{1}$ with $S_{1}$. For the proton, the parameters $f_{T q}^{p}$ are evaluated as



$f_{T u}^{p}=0.020 \pm 0.004, f_{T d}^{p}=0.026 \pm 0.005$ and $f_{T s}^{p}=$ $0.118 \pm 0.062$ [77]. Figure 6 shows the numerical results for direct detection. It is obvious that the predicted $\sigma^{\mathrm{SI}}$ with $\sin \alpha=0.1$ lies below current XENON1T limit, but the range of $M_{N_{1}} \gtrsim 70 \mathrm{GeV}$ is within future LZ's reach. However, if no direct detection signal is observed by LZ, then $\sin \alpha \lesssim 0.02$ should be satisfied.

In Fig. 7, we show the combined results from LFV, $\left|\Delta a_{\mu}\right|$, relic density and LHC search. In left pattern of Fig. 7, it indicates that for $M_{N_{1}}=200 \mathrm{GeV}$, the only exclusion region is from LHC search. Hence, either $M_{\eta} \lesssim 300 \mathrm{GeV}$ with $h_{\mu 1} \lesssim 0.9$ or $M_{\eta} \gtrsim 500 \mathrm{GeV}$ with $h_{\mu 1} \gtrsim 1.3$ is required. In right pattern of Fig. 7, two benchmark value $h_{\mu 1}=1.0,1.5$ are chosen to illustrate. For $h_{\mu 1}=1.0$, we have $250 \mathrm{GeV} \lesssim$ $M_{N_{1}} \lesssim M_{\eta} \sim 400 \mathrm{GeV}$. Therefore, the only viable region is $M_{N_{1}} \sim M_{\eta} \lesssim 400 \mathrm{GeV}$ for $h_{\mu 1} \lesssim 1$. Meanwhile for $h_{\mu 1}=1.5, M_{N_{1}} \gtrsim 120 \mathrm{GeV}$ with $M_{\eta} \gtrsim 520 \mathrm{GeV}$ is able to escape LHC limit.

\subsection{Collider signature}

In this part, we highlight some interesting collider signatures. Begin with the newly discovered $125 \mathrm{GeV}$ Higgs boson $h$ [80,81]. The existence of Majoron $J$ will induce the invisible decay of SM Higgs via $h \rightarrow J J$ [82]. The corresponding decay width is evaluated as

$\Gamma(h \rightarrow J J) \simeq \frac{M_{h}^{3} \sin ^{2} \alpha}{32 \pi v_{S}^{2}}$.

Then, the branching ratio of invisible decay is $\operatorname{BR}(h \rightarrow$ $J J)=\Gamma(h \rightarrow J J) /\left(\Gamma(h \rightarrow J J)+\Gamma_{\mathrm{SM}} \cos ^{2} \alpha\right)$, where $\Gamma_{\mathrm{SM}}=4.09 \mathrm{MeV}$ [83]. Currently, the combined direct

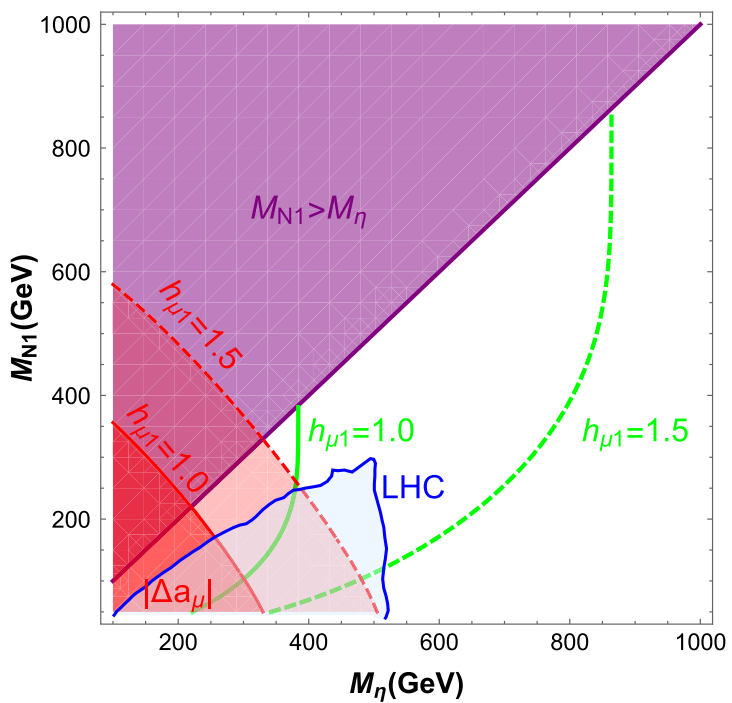

Fig. 7 Combined results for the Yukawa-portal DM. Left pattern: in the $h_{\mu 1}-M_{\eta}$ plane; right pattern: in the $M_{N_{1}}-M_{\eta}$ plane. The green lines satisfy the condition for correct relic density. And the blue regions are excluded by LHC direct search, which will be discussed in Sect. 3.4 



Fig. 8 Branching ratios of scalar singlet $H_{1}$ for $\sin \alpha=0.1$ (left) and $\sin \alpha=0.02$ (right). In this figures, we have also fix $M_{N_{1}}=200 \mathrm{GeV}$ and $v_{S}=10 \mathrm{TeV}$. Note in left pattern, $\operatorname{BR}\left(H_{1} \rightarrow N_{1} N_{1}\right)$ is less than 0.01 , thus is not shown in the plot

and indirect observational limit on invisible Higgs decay is $\mathrm{BR}(h \rightarrow J J)<0.24$ [84]. Typically for $\sin \alpha=0.1, v_{S}=$ $10 \mathrm{TeV}$, we have $\operatorname{BR}(h \rightarrow J J)=4.8 \times 10^{-4}$, which is far below current limit. Meanwhile, if $M_{H_{1}}<2 M_{h}$, then $h \rightarrow H_{1} H_{1}$ with $H_{1} \rightarrow J J$ will also contribute to invisible Higgs decay [85].

In this paper, we consider the high mass scenario $M_{H_{1}}>$ $M_{h}$. In addition to the usual $H_{1} \rightarrow$ SM final states as real singlet model [86], the heavy scalar singlet can also decay into Majoron pair $H_{1} \rightarrow J J$ and DM pair $H_{1} \rightarrow N_{1} N_{1}$. Fig. 8 shows the dominant decay branching ratios of $H_{1}$. The invisible $\mathrm{BR}\left(H_{1} \rightarrow J J\right)$ is less than 0.02 when $\sin \alpha=0.1$, therefore $H_{1}$ appears as a SM heavy Higgs with $\mathrm{BR}\left(H_{1} \rightarrow\right.$ $h h) \approx \mathrm{BR}(h \rightarrow Z Z) \approx \frac{1}{2} \mathrm{BR}\left(H_{1} \rightarrow W W\right) \approx \frac{1}{4}$. While for $\sin \alpha=0.02$, the invisible $\mathrm{BR}\left(H_{1} \rightarrow J J\right)$ increases to about 0.25 , reaching the same order of visible $V V, h h$ decay. And the other invisible decay $H_{1} \rightarrow N_{1} N_{1}$ maximally reaches about 0.03 at $M_{H_{1}} \sim 600 \mathrm{GeV}$. The dominant production channel of $H_{1}$ is via gluon fusion at LHC, which can be estimated as

$\sigma\left(g g \rightarrow H_{1}\right) \approx \sin ^{2} \alpha \times \sigma(g g \rightarrow h)$,

where $\sigma(g g \rightarrow h)$ is the SM Higgs production cross section but calculated with $M_{h}=M_{H_{1}}$. At present, $\sin \alpha \sim 0.1$ [87] leads to the promising signatures as $H_{1} \rightarrow W W \rightarrow e v \mu \nu$ [88], $Z Z \rightarrow 4 \ell$ [89] and $h h \rightarrow 2 b 2 \gamma$ [90,91], etc. In the future, if no DM direct detection signal is observed, then the signature of heavy scalar $H_{1}$ will be much suppressed by tiny value of $\sin \alpha$.

Next, we discuss the gauge boson $Z^{\prime}$ associated with

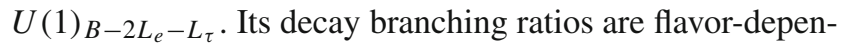
dent, which makes it quite easy to distinguish from the flavoruniversal ones, such as $Z^{\prime}$ from $U(1)_{B-L}$ [92]. Considering

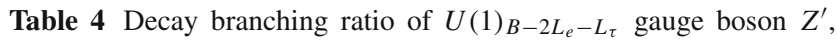
where we have show the lepton flavor individually

\begin{tabular}{lcccccc}
\hline$q \bar{q}$ & $e^{+} e^{-}$ & $\mu^{+} \mu^{-}$ & $\tau^{+} \tau^{-}$ & $\nu \nu$ & $N N$ & $H_{1} H_{1}$ \\
\hline 0.154 & 0.308 & 0 & 0.077 & 0.192 & 0.192 & 0.077 \\
\hline
\end{tabular}

the heavy $Z^{\prime}$ limit, its partial decay width into fermion and scalar pairs are given by

$$
\begin{aligned}
\Gamma\left(Z^{\prime} \rightarrow f \bar{f}\right) & =\frac{M_{Z^{\prime}}}{24 \pi} g^{\prime 2} N_{C}^{f}\left(Q_{f L}^{2}+Q_{f R}^{2}\right), \\
\Gamma\left(Z^{\prime} \rightarrow S S^{*}\right) & =\frac{M_{Z^{\prime}}}{48 \pi} g^{\prime 2} Q_{S}^{2},
\end{aligned}
$$

where $N_{C}^{f}$ is the number of colours of the fermion $f$, i.e., $N_{C}^{l, v}=1, N_{C}^{q}=3$, and $Q_{X}$ is the $U(1)_{B-2 L_{e}-L_{\tau}}$ charge of particle $X$. In Table 4, we present the branching ratio of $Z^{\prime}$. The dominant channel is $Z^{\prime} \rightarrow e^{+} e^{-}$with branching ratio of 0.308 , and no $Z^{\prime} \rightarrow \mu^{+} \mu^{-}$. The $B-2 L_{e}-L_{\tau}$ nature of $Z^{\prime}$ predicts definite relation between quark and lepton final states, e.g.,

$$
\begin{aligned}
& \mathrm{BR}\left(Z^{\prime} \rightarrow b \bar{b}\right): \operatorname{BR}\left(Z^{\prime} \rightarrow e^{+} e^{-}\right) \\
& \quad: \operatorname{BR}\left(Z^{\prime} \rightarrow \mu^{+} \mu^{-}\right): \operatorname{BR}\left(Z^{\prime} \rightarrow \tau^{+} \tau^{-}\right)=\frac{1}{3}: 4: 0: 1,
\end{aligned}
$$

which is also an intrinsic property to distinguish $Z^{\prime}$ of $U(1)_{B-2 L_{e}-L_{\tau}}$ from other flavored gauge bosons [93].

In the framework of $U(1)_{B-2 L_{e}-L_{\tau}}$, one important constraint on $Z^{\prime}$ comes from the precise measurement of fourfermion interactions at LEP [94], which requires

$$
\frac{M_{Z^{\prime}}}{g^{\prime}} \gtrsim 7 \mathrm{TeV} \text {. }
$$



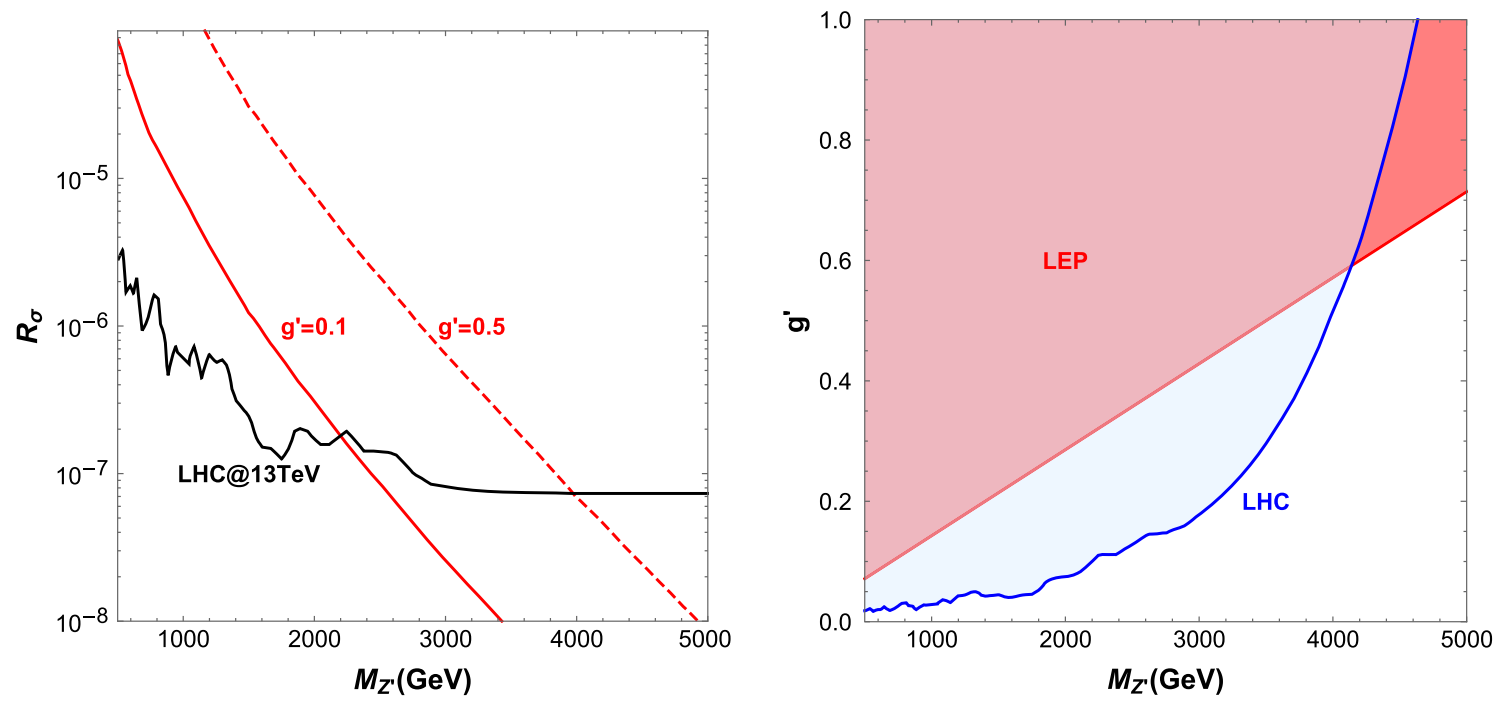

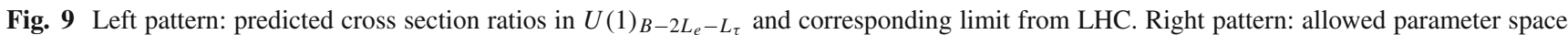
in the $g^{\prime}-M_{Z^{\prime}}$ plane

Since $Z^{\prime}$ couples to both quarks and leptons, the most promising signature at LHC is the dilepton signature $p p \rightarrow Z^{\prime} \rightarrow$ $e^{+} e^{-}$. Searches for such dilepton signature have been performed by ATLAS [95,96] and CMS collaboration [97]. Because of no $Z^{\prime} \rightarrow \mu^{+} \mu^{-}$channel, we can only take the results from CMS, which provides a limit on the ratio

$R_{\sigma}=\frac{\sigma\left(p p \rightarrow Z^{\prime}+X \rightarrow e^{+} e^{-}+X\right)}{\sigma\left(p p \rightarrow Z+X \rightarrow e^{+} e^{-}+X\right)}$.

The theoretical cross section of the dilepton signature are calculated by using MadGraph5_aMC@NLO [98]. Left pattern of Fig. 9 shows that the dilepton signature has excluded $M_{Z^{\prime}} \lesssim 2.2(4.0) \mathrm{TeV}$ for $g^{\prime}=0.1(0.5)$. Then comparing the theoretical ratio with experimental limit, one can acquire the exclusion limit in the $g^{\prime}-M_{Z^{\prime}}$ plane as shown in right pattern of Fig. 9. Obviously, LHC limit is more stringent than LEP when $M_{Z^{\prime}} \lesssim 4 \mathrm{TeV}$.

The inert charge scalars $\eta_{1,2}^{ \pm}$are also observable at LHC. They can decay into charged leptons and right-hand singlets via the Yukawa interactions as

$\Gamma\left(\eta_{1}^{ \pm} \rightarrow \ell^{ \pm} N_{i}\right)=\frac{M_{\eta_{1}^{ \pm}}}{16 \pi}\left|h_{\ell i}^{\prime}\right|^{2}\left(1-\frac{M_{N_{i}}^{2}}{M_{\eta_{1}^{ \pm}}^{2}}\right)^{2}$,

$\Gamma\left(\eta_{2}^{ \pm} \rightarrow \ell^{ \pm} N_{i}\right)=\frac{M_{\eta_{1}^{ \pm}}}{16 \pi}\left|f_{\ell i}^{\prime}\right|^{2}\left(1-\frac{M_{N_{i}}^{2}}{M_{\eta_{2}^{ \pm}}^{2}}\right)^{2}$.

From Eq. (9), we aware that $\eta_{1}^{ \pm}$decays into $\mu, \tau$ final states, while $\eta_{2}^{ \pm}$decays into $e, \tau$ final states. The electron-phobic nature of $\eta_{1}^{ \pm}$and muon-phobic nature of $\eta_{2}^{ \pm}$make them quite easy to distinguish. Meanwhile, their decay branching ratios are related by neutrino oscillation data through the Yukawa coupling $h^{\prime}, f^{\prime}$. Considering the benchmark point in
Table 5 Branching ratios of charge scalar $\eta_{1,2}^{ \pm}$

\begin{tabular}{lllllll}
\hline Final state & $e^{ \pm} N_{1}$ & $\mu^{ \pm} N_{1}$ & $\tau^{ \pm} N_{1}$ & $e^{ \pm} N_{2}$ & $\mu^{ \pm} N_{2}$ & $\tau^{ \pm} N_{2}$ \\
\hline$\eta_{1}^{ \pm}$ & 0.000 & 0.465 & 0.099 & 0.000 & 0.178 & 0.258 \\
$\eta_{2}^{ \pm}$ & 0.043 & 0.000 & 0.611 & 0.113 & 0.000 & 0.233 \\
\hline
\end{tabular}

Eq. (15), the predicted branching ratios are shown in Table 5 in the heavy scalar limit. The dominant decay channel of $\eta_{1}^{ \pm}$is $\mu^{ \pm} N_{1}$, and $\tau^{ \pm} N_{1}$ for $\eta_{2}^{ \pm}$. So $\eta_{1}^{ \pm}$is expected easier to be discovered. Produced via the Drell-Yan process $p p \rightarrow \eta_{1}^{+} \eta_{1}^{-}, \eta_{2}^{+} \eta_{2}^{-}$, the decay channel $\eta_{1,2}^{ \pm} \rightarrow \ell^{ \pm} N_{1}$ then leads to signature $\ell^{+} \ell^{-}+E_{T}$. Exclusion region by direct LHC search for such signature [99] has been shown in right pattern of Fig. 7. To satisfy the direct LHC search bounds, one needs either $M_{N_{1}} \lesssim M_{\eta}<500 \mathrm{GeV}$ or $M_{\eta}>500 \mathrm{GeV}$.

\section{Conclusion}

The scotogenic model is an elegant pathway to explain the origin of neutrino mass and dark matter. Meanwhile, texturezeros in neutrino mass matrix provide a promising way to under stand the leptonic flavor structure. Therefore, it is appealing to connect the scotogenic model with texturezeros. In this paper, we propose a viable approach to realise two texture-zeros in the scotogenic model with flavor dependent $U(1)_{B-2 L_{\alpha}-L_{\beta}}$ gauge symmetry. These models are extended by two right-handed singlets $N_{R i}$ and two inert scalar doublets $\eta_{i}$, which are odd under the dark $Z_{2}$ symmetry. Six kinds of texture-zeros are realised in our approach, i.e., texture $A_{1}, A_{2}, B_{3}, B_{4}, D_{1}$ and $D_{2}$. Among all the six 
texture-zeros, we find that texture $A_{1}$ and $A_{2}$ are allowed by current experimental limits, while texture $B_{3}$ and $B_{4}$ are marginally allowed. Besides, texture $D_{1}$ and $D_{2}$ are already excluded by neutrino oscillation data.

Realization of texture-zeros in the scotogenic model makes the model quite predictive. And we have taken texture $A_{1}$ derived from $U(1)_{B-2 L_{e}-L_{\tau}}$ for illustration. Some distinct features are summarized in the following:

- The texture $A_{1}$ predicts vanishing neutrinoless double beta decay rate. And only normal neutrino mass hierarchy is allowed. It predicts $m_{1} \sim 0.007 \mathrm{eV}, m_{2} \sim 0.01 \mathrm{eV}$, and $m_{3} \approx \sqrt{\Delta m^{2}} \sim 0.05 \mathrm{eV}$, then $\sum m_{i} \sim 0.07 \mathrm{eV}$. There are also strong correlation between the Dirac and Majorana phases, i.e., $\rho \approx \frac{\delta}{2}$ and $\sigma \approx \frac{\delta}{2}-\frac{\pi}{2}$.

- The ratios of corresponding Yukawa couplings are also predicted by neutrino oscillation data, e.g.,

$\frac{h_{\tau 2}}{h_{\mu 1}}: \frac{f_{\tau 1}}{h_{\mu 1}}: \frac{f_{e 2}}{h_{\mu 1}} \simeq 0.745: 0.933: 0.401$.

- Due to specific Yukawa structure, the LFV process $\mu \rightarrow$ $e \gamma$ is missing at one-loop level. Meanwhile, large cancellations are possible for $\tau \rightarrow \mu \gamma$ and $\tau \rightarrow e \gamma$ with degenerate right-handed singlets. More stringent constraint comes from muon anomalous magnetic moment $\Delta a_{\mu}$. Although $\mathcal{O}(1)$ Yukawa couplings are easily to avoid such limit.

- Satisfying all constraints, correct relic density of dark matter $N_{1}$ is achieved for $M_{N_{1}} \lesssim M_{\eta}<500 \mathrm{GeV}$ with $h_{\mu 1} \lesssim 1$ or $M_{\eta}>500 \mathrm{GeV}$ with $h_{\mu 1}>1$.As for direct detection, we have shown that the predicted spin-independent DM-nucleon cross section $\sigma^{\text {SI }}$ with $\sin \alpha=0.1$ satisfies the current XENON1T limit, but is within future reach of LZ.

- The Majoron $J$ contributes to invisible decay of SM Higgs. The additional scalar singlet $H_{1}$ can be probe in the channel $g g \rightarrow H_{1} \rightarrow W^{+} W^{-}, Z Z$ at LHC. Decays of charged scalars $\eta_{1,2}^{ \pm}$lead to $p p \rightarrow \eta_{1,2}^{+} \eta_{1,2}^{-} \rightarrow \ell^{+} \ell^{-}+$ $E_{T}$ signature. Note that the corresponding branching ratios are also correlated with neutrino oscillation parameters.

- The neutral gauge boson $Z^{\prime}$ is promising via the dielectron signature $p p \rightarrow Z^{\prime} \rightarrow e^{+} e^{-}$. Its $B-2 L_{e}-L_{\tau}$ nature can be confirmed by

$$
\begin{aligned}
& \operatorname{BR}\left(Z^{\prime} \rightarrow b \bar{b}\right): \operatorname{BR}\left(Z^{\prime} \rightarrow e^{+} e^{-}\right): \operatorname{BR}\left(Z^{\prime} \rightarrow \mu^{+} \mu^{-}\right) \\
& \quad: \operatorname{BR}\left(Z^{\prime} \rightarrow \tau^{+} \tau^{-}\right)=\frac{1}{3}: 4: 0: 1
\end{aligned}
$$

In a nutshell, the scotogenic model with flavor dependent $U(1)_{B-2 L_{\alpha}-L_{\beta}}$ symmetry predicts distinct and observable phenomenology, which is useful to distinguish from other models.

Acknowledgements The work of Weijian Wang is supported by National Natural Science Foundation of China under Grant Numbers 11505062, Special Fund of Theoretical Physics under Grant Numbers 11447117 and Fundamental Research Funds for the Central Universities under Grant Numbers 2014ZD42. The work of Zhi-Long Han is supported by National Natural Science Foundation of China under Grant Nos. 11805081 and 11605075, Natural Science Foundation of Shandong Province under Grant Nos. ZR2019QA021, ZR2018MA047, ZR2017JL006 and ZR2014AM016.

Data Availability Statement This manuscript has no associated data or the data will not be deposited. [Authors' comment: The data can be directly read from the figures.]

Open Access This article is distributed under the terms of the Creative Commons Attribution 4.0 International License (http://creativecomm ons.org/licenses/by/4.0/), which permits unrestricted use, distribution, and reproduction in any medium, provided you give appropriate credit to the original author(s) and the source, provide a link to the Creative Commons license, and indicate if changes were made.

Funded by SCOAP $^{3}$.

\section{References}

1. E. Ma, Phys. Rev. D 73, 077301 (2006). arXiv:hep-ph/0601225

2. L.M. Krauss, S. Nasri, M. Trodden, Phys. Rev. D 67, 085002 (2003). arXiv:hep-ph/0210389

3. M. Aoki, S. Kanemura, O. Seto, Phys. Rev. Lett. 102, 051805 (2009). arXiv:0807.0361 [hep-ph]

4. E. Ma, Mod. Phys. Lett. A 21, 1777 (2006). arXiv:hep-ph/0605180

5. T. Hambye, K. Kannike, E. Ma, M. Raidal, Phys. Rev. D 75, 095003 (2007). arXiv:hep-ph/0609228

6. D. Aristizabal Sierra, J. Kubo, D. Restrepo, D. Suematsu, O. Zapata, Phys. Rev. D 79, 013011 (2009). arXiv:0808.3340 [hep-ph]

7. D. Suematsu, T. Toma, T. Yoshida, Phys. Rev. D 79, 093004 (2009). arXiv:0903.0287 [hep-ph]

8. D. Schmidt, T. Schwetz, T. Toma, Phys. Rev. D 85, 073009 (2012). arXiv:1201.0906 [hep-ph]

9. R. Bouchand, A. Merle, JHEP 1207, 084 (2012). arXiv: 1205.0008 [hep-ph]

10. A. Merle, M. Platscher, JHEP 1511, 148 (2015). arXiv: 1507.06314 [hep-ph]

11. E. Ma, A. Natale, A. Rashed, Int. J. Mod. Phys. A 27, 1250134 (2012). arXiv:1206.1570 [hep-ph]

12. M. Klasen, C.E. Yaguna, J.D. Ruiz-Alvarez, D. Restrepo, O. Zapata, JCAP 1304, 044 (2013). arXiv:1302.5298 [hep-ph]

13. S.Y. Ho, J. Tandean, Phys. Rev. D 87, 095015 (2013). arXiv: 1303.5700 [hep-ph]

14. A. Ahriche, S. Nasri, JCAP 1307, 035 (2013). arXiv:1304.2055 [hep-ph]

15. M. Chekkal, A. Ahriche, A.B. Hammou, S. Nasri, Phys. Rev. D 95(9), 095025 (2017). arXiv:1702.04399 [hep-ph]

16. K.P. Modak, JHEP 1503, 064 (2015). arXiv:1404.3676 [hep-ph]

17. E. Molinaro, C.E. Yaguna, O. Zapata, JCAP 1407, 015 (2014). arXiv:1405.1259 [hep-ph]

18. G. Faisel, S.Y. Ho, J. Tandean, Phys. Lett. B 738, 380 (2014). arXiv: 1408.5887 [hep-ph]

19. A. Merle, M. Platscher, Phys. Rev. D 92(9), 095002 (2015). arXiv:1502.03098 [hep-ph] 
20. A. Ahriche, K.L. McDonald, S. Nasri, JHEP 1602, 038 (2016). arXiv:1508.02607 [hep-ph]

21. A. Ahriche, K.L. McDonald, S. Nasri, JHEP 1606, 182 (2016). arXiv:1604.05569 [hep-ph]

22. A. Ahriche, A. Manning, K.L. McDonald, S. Nasri, Phys. Rev. D 94(5), 053005 (2016). arXiv:1604.05995 [hep-ph]

23. M. Lindner, M. Platscher, C.E. Yaguna, A. Merle, Phys. Rev. D 94(11), 115027 (2016). arXiv:1608.00577 [hep-ph]

24. A.G. Hessler, A. Ibarra, E. Molinaro, S. Vogl, JHEP 1701, 100 (2017). arXiv:1611.09540 [hep-ph]

25. D. Borah, A. Gupta, Phys. Rev. D 96(11), 115012 (2017). arXiv:1706.05034 [hep-ph]

26. A. Abada, T. Toma, JHEP 1804, 030 (2018). arXiv:1802.00007 [hep-ph]

27. T. Hugle, M. Platscher, K. Schmitz, Phys. Rev. D 98(2), 023020 (2018). arXiv:1804.09660 [hep-ph]

28. S. Baumholzer, V. Brdar, P. Schwaller, JHEP 1808, 067 (2018). arXiv:1806.06864 [hep-ph]

29. D. Borah, P.S.B. Dev, A. Kumar, arXiv:1810.03645 [hep-ph]

30. L. Bian, X. Liu, arXiv:1811.03279 [hep-ph]

31. E. Ma, I. Picek, B. Radovčič, Phys. Lett. B 726, 744 (2013). arXiv: 1308.5313 [hep-ph]

32. Y. Cai, J. Herrero-García, M.A. Schmidt, A. Vicente, R.R. Volkas, Front. Phys. 5, 63 (2017). arXiv:1706.08524 [hep-ph]

33. P.H. Frampton, S.L. Glashow, D. Marfatia, Phys. Lett. B 536, 79 (2002). arXiv:hep-ph/0201008]

34. H. Fritzsch, Z.Z. Xing, JHEP 1109, 083 (2011). arXiv:1108.4534 [hep-ph]

35. J. Alcaide, J. Salvado, A. Santamaria, JHEP 1807, 164 (2018). arXiv:1806.06785 [hep-ph]

36. W. Grimus, A.S. Joshipura, L. Lavoura, M. Tanimoto, Eur. Phys. J. C 36, 227 (2004). arXiv:hep-ph/0405016

37. T. Araki, J. Heeck, J. Kubo, JHEP 1207, 083 (2012). arXiv:1203.4951 [hep-ph]

38. L.M. Cebola, D. Emmanuel-Costa, R. Gonzalez Felipe, Phys. Rev. D 88(11), 116008 (2013). arXiv:1309.1709 [hep-ph]

39. T. Kitabayashi, Phys. Rev. D 98(8), 083011 (2018). arXiv:1808.01060 [hep-ph]

40. T. Kitabayashi, S. Ohkawa, M. Yasuè, Int. J. Mod. Phys. A 32(32), 1750186 (2017). arXiv: 1703.09417 [hep-ph]

41. S. Baek, H. Okada, K. Yagyu, JHEP 1504, 049 (2015). arXiv:1501.01530 [hep-ph]

42. S. Baek, Phys. Lett. B 756, 1 (2016). arXiv:1510.02168 [hep-ph]

43. S. Lee, T. Nomura, H. Okada, Nucl. Phys. B 931, 179 (2018). arXiv:1702.03733 [hep-ph]

44. K. Asai, K. Hamaguchi, N. Nagata, S.Y. Tseng, K. Tsumura, arXiv:1811.07571 [hep-ph]

45. T. Nomura, H. Okada, Phys. Dark Univ. 21, 90 (2018). arXiv:1712.00941 [hep-ph]

46. P. Ko, T. Nomura, H. Okada, Phys. Lett. B 772, 547 (2017). arXiv:1701.05788 [hep-ph]

47. T. Nomura, H. Okada, arXiv:1806.09957 [hep-ph]

48. N. Aghanim et al. [Planck Collaboration], arXiv:1807.06209 [astro-ph.CO]

49. I. Esteban, M.C. Gonzalez-Garcia, A. Hernandez-Cabezudo, M. Maltoni, T. Schwetz, arXiv:1811.05487 [hep-ph]

50. E.I. Lashin, N. Chamoun, Phys. Rev. D 85, 113011 (2012). arXiv: 1108.4010 [hep-ph]

51. A. Vicente, C.E. Yaguna, JHEP 1502, 144 (2015). arXiv: 1412.2545 [hep-ph]

52. S.M. Bilenky, C. Giunti, W. Grimus, B. Kayser, S.T. Petcov, Phys. Lett. B 465, 193 (1999). arXiv:hep-ph/9907234]

53. F. Vissani, JHEP 9906, 022 (1999). arXiv:hep-ph/9906525]

54. S. Vagnozzi, E. Giusarma, O. Mena, K. Freese, M. Gerbino, S. Ho, M. Lattanzi, Phys. Rev. D 96(12), 123503 (2017). arXiv:1701.08172 [astro-ph.CO]
55. T. Toma, A. Vicente, JHEP 1401, 160 (2014). arXiv:1312.2840 [hep-ph]

56. R. Ding, Z.L. Han, Y. Liao, H.J. Liu, J.Y. Liu, Phys. Rev. D 89(11), 115024 (2014). arXiv:1403.2040 [hep-ph]

57. B. Aubert et al., BaBar Collaboration, Phys. Rev. Lett. 104, 021802 (2010). arXiv:0908.2381 [hep-ex]

58. K. Hayasaka [Belle and Belle-II Collaborations], J. Phys. Conf. Ser. 408, 012069 (2013)

59. M. Lindner, M. Platscher, F.S. Queiroz, Phys. Rept. 731, 1 (2018). arXiv:1610.06587 [hep-ph]

60. T. Blum, A. Denig, I. Logashenko, E. de Rafael, B.L. Roberts, T. Teubner, G. Venanzoni, arXiv:1311.2198 [hep-ph]

61. J. Kubo, E. Ma, D. Suematsu, Phys. Lett. B 642, 18 (2006). arXiv: hep-ph/0604114

62. T. Li, W. Chao, Nucl. Phys. B 843, 396 (2011). arXiv:1004.0296 [hep-ph]

63. G. Bertone, D. Hooper, J. Silk, Phys. Rept. 405, 279 (2005). arXiv:hep-ph/0404175]

64. N. Okada, O. Seto, Phys. Rev. D 82, 023507 (2010). arXiv: 1002.2525 [hep-ph]

65. S. Kanemura, O. Seto, T. Shimomura, Phys. Rev. D 84, 016004 (2011). arXiv:1101.5713 [hep-ph]

66. N. Okada, Y. Orikasa, Phys. Rev. D 85, 115006 (2012) arXiv:1202.1405 [hep-ph]

67. W. Wang, Z.L. Han, Phys. Rev. D 92, 095001 (2015). arXiv:1508.00706 [hep-ph]

68. N. Okada, S. Okada, Phys. Rev. D 93(7), 075003 (2016). arXiv:1601.07526 [hep-ph]

69. N. Okada, S. Okada, D. Raut, Phys. Lett. B 780, 422 (2018). arXiv:1712.05290 [hep-ph]

70. Z.L. Han, W. Wang, R. Ding, Eur. Phys. J. C 78(3), 216 (2018). arXiv:1712.05722 [hep-ph]

71. S. Okada, Adv. High Energy Phys. 2018, 5340935 (2018). arXiv:1803.06793 [hep-ph]

72. Z.L. Han, W. Wang, Eur. Phys. J. C 78(10), 839 (2018). arXiv:1805.02025 [hep-ph]

73. N. Okada, S. Okada, D. Raut, arXiv:1811.11927 [hep-ph]

74. D. Borah, D. Nanda, N. Narendra, N. Sahu, arXiv:1810.12920 [hep-ph]

75. Y.G. Kim, K.Y. Lee, C.B. Park, S. Shin, Phys. Rev. D 93(7), 075023 (2016). arXiv:1601.05089 [hep-ph]

76. R. Ding, Z.L. Han, L. Huang, Y. Liao, Chin. Phys. C 42(10), 103101 (2018). arXiv:1802.05248 [hep-ph]

77. J.R. Ellis, A. Ferstl, K.A. Olive, Phys. Lett. B 481, 304 (2000). arXiv:hep-ph/0001005

78. E. Aprile et al. [XENON Collaboration], Phys. Rev. Lett. 121(11), 111302 (2018). arXiv:1805.12562 [astro-ph.CO]

79. D.S. Akerib et al. [LUX-ZEPLIN Collaboration], arXiv:1802.06039 [astro-ph.IM]

80. G. Aad et al., ATLAS Collaboration, Phys. Lett. B 716, 1 (2012). arXiv:1207.7214 [hep-ex]

81. S. Chatrchyan et al., CMS Collaboration, Phys. Lett. B 716, 30 (2012). arXiv:1207.7235 [hep-ex]

82. W. Wang, Z.L. Han, Phys. Rev. D 94(5), 053015 (2016). arXiv:1605.00239 [hep-ph]

83. D. de Florian et al. [LHC Higgs Cross Section Working Group], arXiv:1610.07922 [hep-ph]

84. V. Khachatryan et al., CMS Collaboration, JHEP 1702, 135 (2017). arXiv:1610.09218 [hep-ex]

85. C. Bonilla, J.W.F. Valle, J.C. Romão, Phys. Rev. D 91(11), 113015 (2015). arXiv:1502.01649 [hep-ph]

86. T. Robens, T. Stefaniak, Eur. Phys. J. C 76(5), 268 (2016). arXiv:1601.07880 [hep-ph]

87. A. Ilnicka, T. Robens, T. Stefaniak, Mod. Phys. Lett. A 33(10-11), 1830007 (2018). arXiv:1803.03594 [hep-ph] 
88. M. Aaboud et al. [ATLAS Collaboration], Eur. Phys. J. C 78(1), 24 (2018). arXiv:1710.01123 [hep-ex]

89. M. Aaboud et al. [ATLAS Collaboration], Phys. Rev. D 98(5), 052008 (2018). arXiv:1808.02380 [hep-ex]

90. A.M. Sirunyan et al., CMS Collaboration, Phys. Lett. B 788, 7 (2019). arXiv: 1806.00408 [hep-ex]

91. M. Aaboud et al., ATLAS Collaboration, JHEP 1811, 040 (2018). arXiv:1807.04873 [hep-ex]

92. L. Basso, A. Belyaev, S. Moretti, C.H. Shepherd-Themistocleous, Phys. Rev. D 80, 055030 (2009). arXiv:0812.4313 [hep-ph]

93. E.J. Chun, A. Das, J. Kim, J. Kim, arXiv:1811.04320 [hep-ph]
94. G. Cacciapaglia, C. Csaki, G. Marandella, A. Strumia, Phys. Rev. D 74, 033011 (2006). arXiv:hep-ph/0604111

95. M. Aaboud et al., ATLAS Collaboration, JHEP 1710, 182 (2017). arXiv:1707.02424 [hep-ex]

96. G. Aad et al. [ATLAS Collaboration], arXiv:1903.06248 [hep-ex]

97. A.M. Sirunyan et al., CMS Collaboration, JHEP 1806, 120 (2018). arXiv:1803.06292 [hep-ex]

98. J. Alwall et al., JHEP 1407, 079 (2014). arXiv:1405.0301 [hep-ph]

99. M. Aaboud et al. [ATLAS Collaboration], Eur. Phys. J. C 78(12), 995 (2018). arXiv: 1803.02762 [hep-ex] 